\title{
Presentation
}

\section{Nodding Syndrome in Uganda: Field Observations, Challenges and Research Agenda}

\author{
Hermann Feldmeier ${ }^{1 *}$, Osuke Komazawa ${ }^{2}$ and Kazuhiko Moji ${ }^{3}$ \\ Published online 27 May, 2014
}

\begin{abstract}
This article summarizes observations made in Northern Uganda and a lecture given at the Nodding Syndrome Workshop in Nagasaki September 2013. The objective of the manuscript is to summarize the current knowledge on nodding syndrome and to provide an agenda for investigations into the epidemiology, pathophysiology, diagnosis and care management of nodding syndrome in Uganda.
\end{abstract}

Key words: Nodding syndrome, Uganda

\section{Historical AsPects}

In the 1950s the Norwegian psychiatrist Louise JilekAall reported an hitherto unknown neurologic disease while working in the southern part of Tanzania in an area inhabited by the Pogoro people [1]. She noticed a peculiar form of epilepsy that started with nodding of the head and in later stages of the disease was accompanied by impaired cognitive functions.

In the mid-1990s health providers from the Equatoria States of the Sudan (an area near to the Ugandan border) reported an increasing number of children with nodding syndrome to the Ministry of Health (MOH) in Khartoum. However, only in 2001 Sudanese health officials notified the emerging epidemic to WHO.

Similarly, in the mid-2000s health workers and local NGOs from the Northern District of Uganda reported increasing numbers of children with nodding syndrome to the MOH in Kampala. Only in 2011 the MOH sent a team to the area to collect data on the occurrence of nodding syndrome in Kitgum, Lamwo and Pader district.

\section{DiseAse OCCURRENCE}

Almost all cases of nodding syndrome notified so far occurred in the Northern Districts of Uganda (Fig. 1) and in several counties of the Western, Central and Eastern Equatoria States of South Sudan (a new nation independent since 2011).
The area is inhabited by the Acholi people. Reliable data on disease occurrence do not exist and only estimates are at hand (Table 1).

The heterogeneous spatial distribution of patients with nodding syndrome within the endemic area is striking (Table 2).

There is a considerable spatial overlap between nodding syndrome and epilepsy sui generis (Table 3 ).

Supposedly, part of the cases recorded as epilepsy are the patients with nodding syndrome in whom the disease started with (grand mal or other type) seizures and who had not yet developed nodding attacks. Since 2000, notifications have increased considerably (Fig. 2a, b, c).

No data are available beyond 2010. Incidence data (i.e. onset of disease per 10,000 children per year) are not available.

The age of onset peaks between 8 and 15 years (Fig. 3). 95\% of the patients developed the disease between 5 and 16 years.

\section{Clinical Neurology}

In about half of the patients the disease starts with the characteristic nodding of the head (Table 4).

About one third of the parents note an abnormal behavior of the child as a first sign. The remaining cases begin with classical epilepsy. Nodding may be accompanied by drooling of saliva, urine incontinence and other neurological symptoms. Cognitive function impairment

\footnotetext{
${ }^{1}$ Institute for Microbiology and Hygiene, Charité University Medicine, Berlin Campus Benjamin Franklin, Hindenburgdamm 3012203 Berlin, Germany

${ }^{2}$ Nagasaki University Hospital, 1-7-1 Sakamoto, Nagasaki, 8528501, Japan

${ }^{3}$ Nagasaki University Graduate School of International Health Development, 1-12-4 Sakamoto, Nagasaki, 8528523, Japan

*Corresponding author:

Tel: +49-163-674 3707

E-mail: Feldmeier.FUBerlin@t-online.de
} 


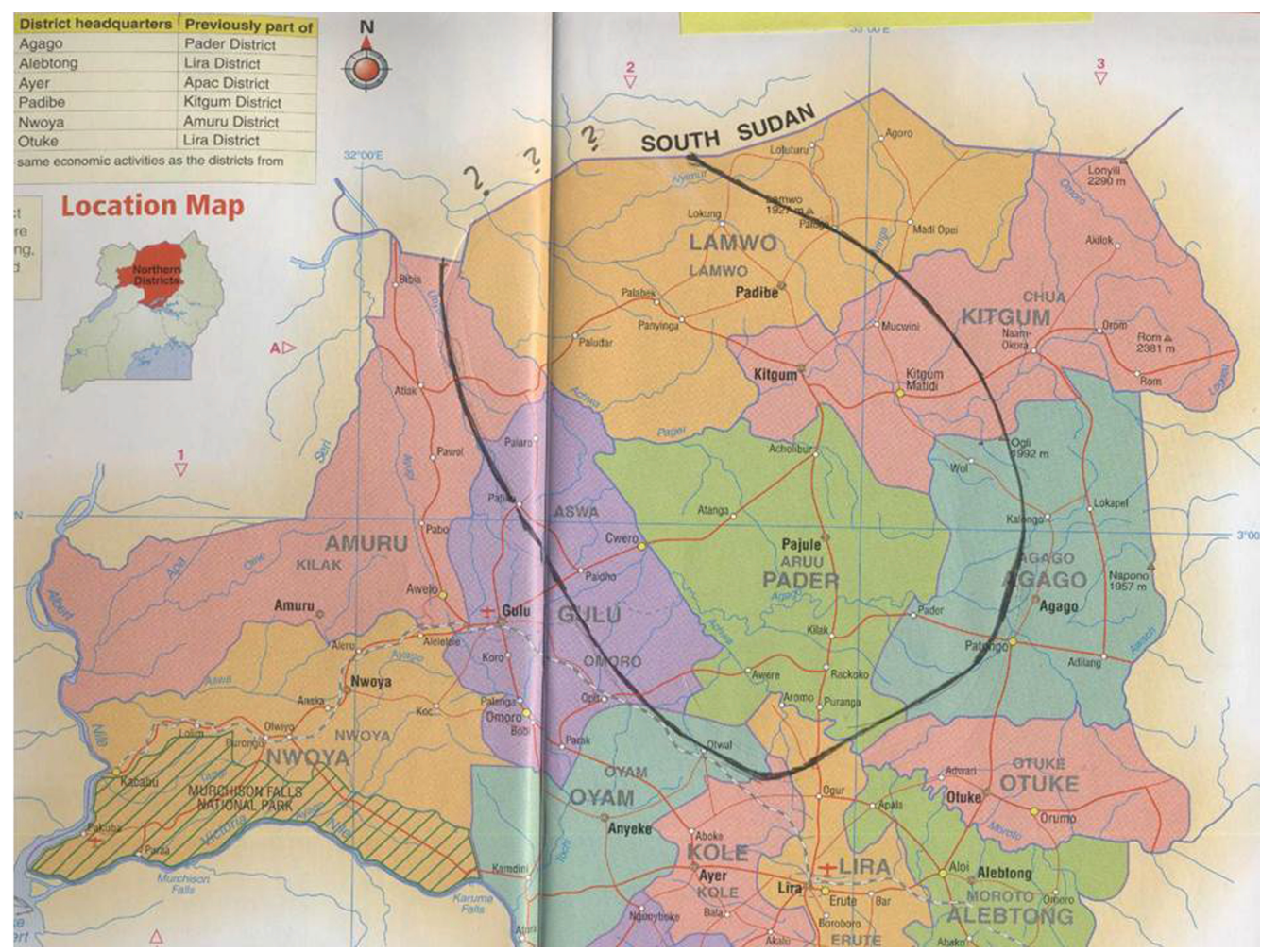

Fig. 1. Area affected by nodding syndrome in Northern Uganda

Table 1. Dimension of the problem

\begin{tabular}{|c|c|}
\hline Uganda & \\
\hline Pader+ Lamwo+Kitgum district* & $\approx 2800$ cases; ? dead \\
\hline Pader district** & $\approx 5000$ cases; 300 dead \\
\hline \multicolumn{2}{|l|}{ South Sudan } \\
\hline $\begin{array}{l}\text { Counties of Western, Central, } \\
\text { Eastern Equatoria states*** }\end{array}$ & $\approx 5000-8000$ cases; ? dead \\
\hline
\end{tabular}

develops over time. In a small case series generalized cerebral atrophy was detected in MRI (Table 5) [2].

Hitherto, no longitudinal studies have been performed and the natural history of the disease remains enigmatic. How many patients die, and why death occurs are not known (Table 6).

\section{SOCIAL AND ECONOMIC IMPLICATIONS}

Nodding syndrome has a devastating effect on families and communities (Table 7).
Table 2. Heterogenous distribution of cases with foci and clusters in Uganda

\begin{tabular}{ll}
\hline District & Prevalence /100,000 \\
\hline Kitgum & 188 \\
Lamwo & 232 \\
Pader & 519 \\
\hline & \\
Counties of Kitgum district & Prevalence /100,000 \\
\hline Kitgum town & 3 \\
Labongo Layamo & 91 \\
Labongo Amida & 260 \\
Labongo Akwang & 1305 \\
\hline Report MOH Uganda 2010 &
\end{tabular}

Children with nodding syndrome have to be under continuous observation. Those in an advanced stage have to be nursed. Patients and their caretakers are stigmatized. Social exclusion will be the consequence. The situation is aggravated by the fact that the disease often affects several children within one family. 
Table 3. Nodding Syndrome and Epilepsy in Pader District

\begin{tabular}{llll}
\hline & Median & IQR & Range \\
\hline $\begin{array}{l}\text { NS cases } \\
/ 100,000\end{array}$ & 117 & $22-405$ & $4-861$ \\
$\begin{array}{l}\text { NS deaths / } \\
\text { NS cases }\end{array}$ & 0.06 & $0.05-0.09$ & $0.03-0.11$ \\
\hdashline $\begin{array}{l}\text { Epilepsy cases / } \\
\text { 100,000 }\end{array}$ & 358 & $264-592$ & $224-680$ \\
$\begin{array}{l}\text { Epilepsy deaths/ } \\
\text { Epilepsy cases }\end{array}$ & 0.07 & $0.04-0.08$ & $0.03-0.11$ \\
\hline
\end{tabular}

Debriefing Report MOH, July 2012

\section{a Kitgum District}
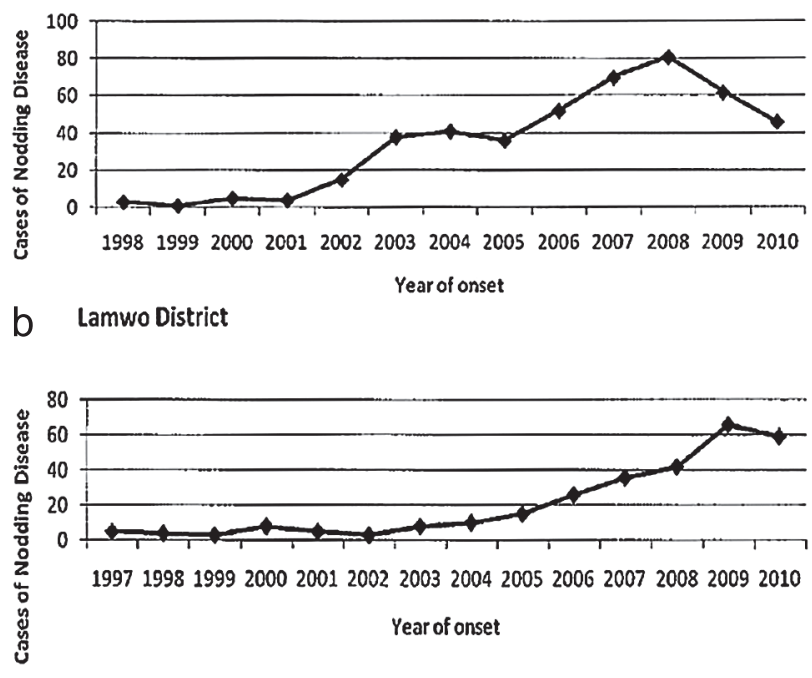

\section{Pader District}

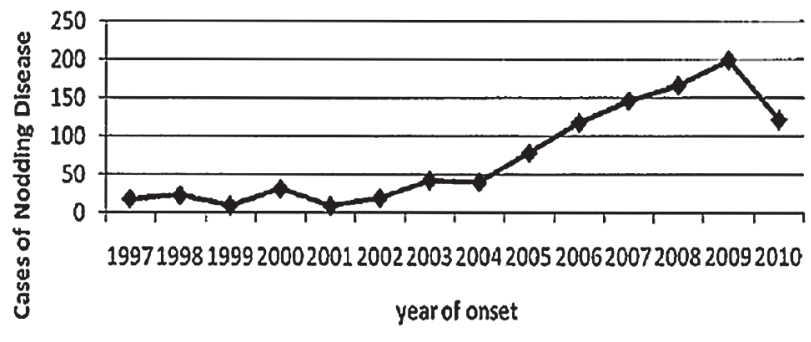

\section{Report MOH Uganda 2010}

Fig. 2. Trend of detected cases in Northern Uganda between 1997 and 2010

\section{The Search for Causes (Table 8)}

Several categories of causes have been proposed. Recently, Dowell et al. (3) summarized the four small case control studies hitherto performed (Table 9).

The authors came to the conclusion that nodding syn-

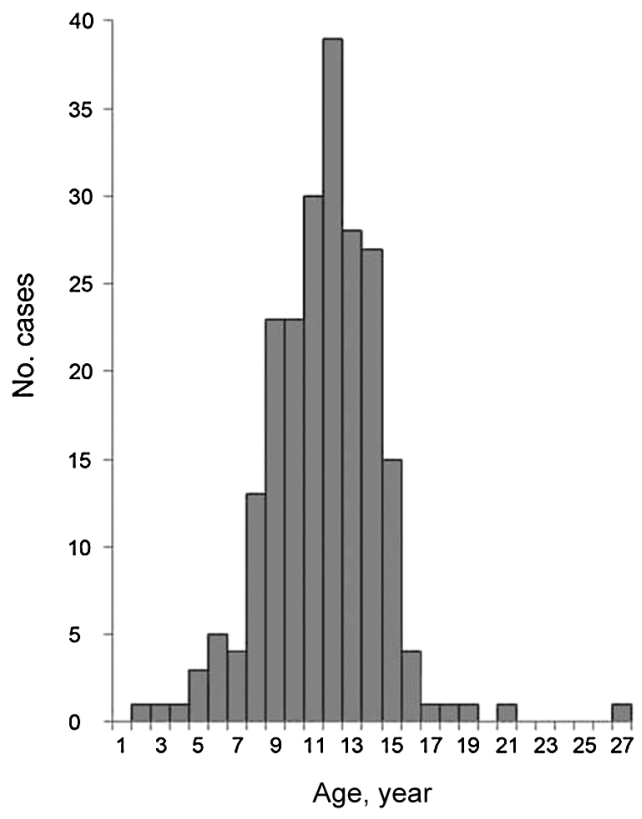

Fig. 3. Number of cases according to age [3]

Table 4. Nodding is a particular manifestation of atonic seizures

- consists of repetitive bobbing or nodding of the head, sometimes associated with loss of muscle tone in the trunk and upper extremities

- often occurs in response to stimuli: food presented, cold air, ...

- \pm loss of consciousness

Table 5. Clinical, neurological, and eloctrophysiological features of nodding syndrome in Kitgum, Uganda: an observational case series

- 14/23 seizures

- $7 / 23$ gross cognitive function impairment

- 23/23 normal glucose+protein in CSF

- 12/12 abnormal EEG indicating atonic seizures

- 4/5 pathological MRI with generalized cerebral and cerebellar atrophy

- $6 / 12$ worsened during 8 months follow-up

$\sum$ Nodding syndrome is a progressive epidemic epilepsy associated with encephalopathy, with head nodding caused by atonic seizures.

Lancet Neurology 2013, 12 (2) 166-174 
Table 6. Putative causes of death

- malnutrition $\longrightarrow$ cachexia?

- prolonged seizures \pm fatal burns, drowning?

- progressive cerebral+cerebellar encephalopathy?

- self-injury?

- others?

Table 7. Nodding syndrome has a devastating effect on families and communities

- previously healthy children drop out of school

- lose ability to eat and cognitive functions

- require constant oversight (strolling around; burning, drowning)

- lose social bonds; patients and families are stigmatized

- the impact on household/community economics is expected to be high

Table 8. The search for cause(s)

- Infectious causes (+ genetic trait):

Parasitic diseases: onchocerciasis, malaria, sleeping sickness, crsticercosis, toxoplasmosis, schistosomiasis, other helminths?

- Bacterial diseases: congenital syphilis; other treponema species?

- Viral diseases: neurotropic virus transmitted by black flies? HTLV; prion?

- Toxic causes: ingestion of toxic substances in food (heavy metals, plant poisons ...?), chemicals from weapons, seed pesticides?

- Autoimmune disorders: viral infections, vaccines?

- Trauma and bad spirits: traumatic events during war; souls of people killed?

drome is an epidemic epilepsy for which the cause remains enigmatic.

Several authors have observed an association between onchocerciasis and nodding syndrome. However, it is difficult to envisage that the infection with Onchocerca volvulus could cause nodding syndrome: onchocerciasis is endemic in many countries of West, Central and East Africa and occurs since a hundred of years. However, nodding syndrome is a recent disease and occurs only in a geographically limited area. We suggest that the nodding syndrome increases the exposure to $O$. volvulus: since the patients become autistic and rather immobile when the dis-
Table 9. Causes of Nodding Syndrome

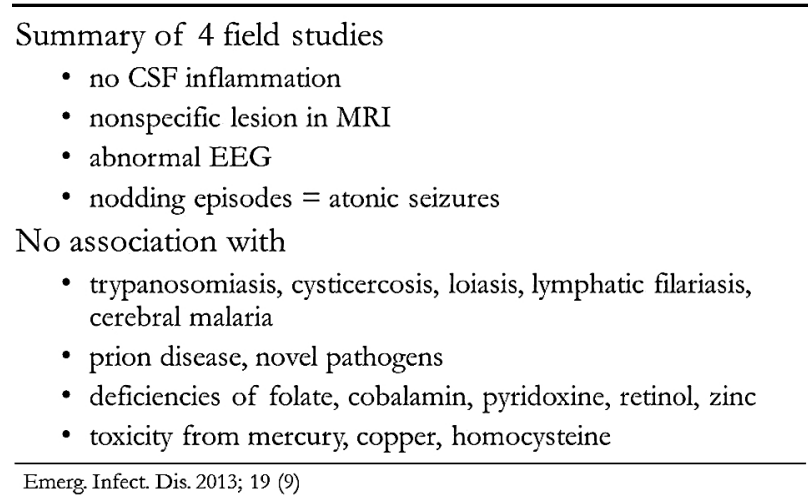

Table 10. Perceived Causes

- Refugee Camps

rotten food: beans, pease, maize, groundnut, durrha; cooking oil (Argentina); milk powder seeds treated with pesticide

contaminated drinking water: chemicals from ammunition, poisoned by LRA

- Transit sites

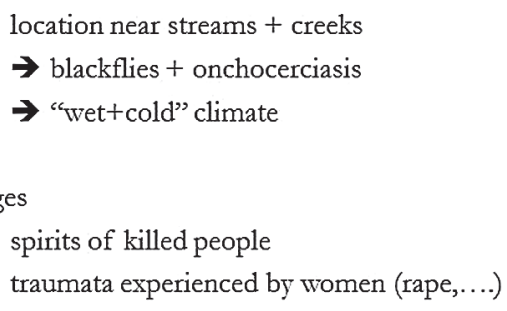

ease progresses, the chances to be bitten by black flies are higher than in healthy children. Hence, nodding syndrome could be the cause of an increased frequency of onchocerciasis in patients with nodding syndrome.

In the interviews conducted by one of our authors (HF), mothers of children with nodding syndrome mentioned trauma experienced during the civil war and the existence of bad spirits as the causes of the disease. However, disease concepts rooted in the history and culture of Acholi people are only imperfectly understood.

Mothers consented that nodding syndrome is linked to their presence in IDP (internally displaced persons) camps into which the Acholi were forced to stray during the civil war (1986-2008) (Table 10).

Hunger was constant in the camps and people had to eat rotten food and pesticide-impregnated seeds (foreseen to be planted and not to be eaten!). However, a rationale 
how the ingestion of toxic substances, such as fungal toxins or pesticides or heavy metal, by the mother can lead to development of nodding syndrome in their children is lacking.

There seems to be a relationship between children conceived/born in camps and the later development of nodding syndrome: out of 64 children seen in May 2013, 11 were born in a IDP camp (or shortly later) and developed nodding syndrome when they were between one and nine years old. Four out of the 15 patients, though, were born in the village before parents moved to a camp (Table 11).

\section{TREATMENT}

Since the pathophysiology of nodding syndrome is not understood, there is no causal treatment. According to the guidelines of the $\mathrm{MOH}$ of Uganda, patients are treated with simultaneously seven anti-epileptic drugs, plus vitamin B6 (Fig. 4). Despite the multidrug treatment mothers

Table 11. Interrogation of 8 families in 4 villages

\begin{tabular}{ll}
\hline $\begin{array}{l}\text { Children born between 1993-2009 } \\
\text { - died at young age (unrelated to NS) }\end{array}$ & 64 \\
- with NS alive & $15(q=7 ; \hat{\jmath}=8)$ \\
- with NS dead & 0 \\
- with NS born in camp & 10 \\
- with NS born in village $\leq 2$ years after & 1 \\
return from camp & 4 \\
- with NS born in village before camp & $1-3$ \\
no of cases in single family & \\
Appearance of first sign: median 5 years (range 1-9 years)
\end{tabular}

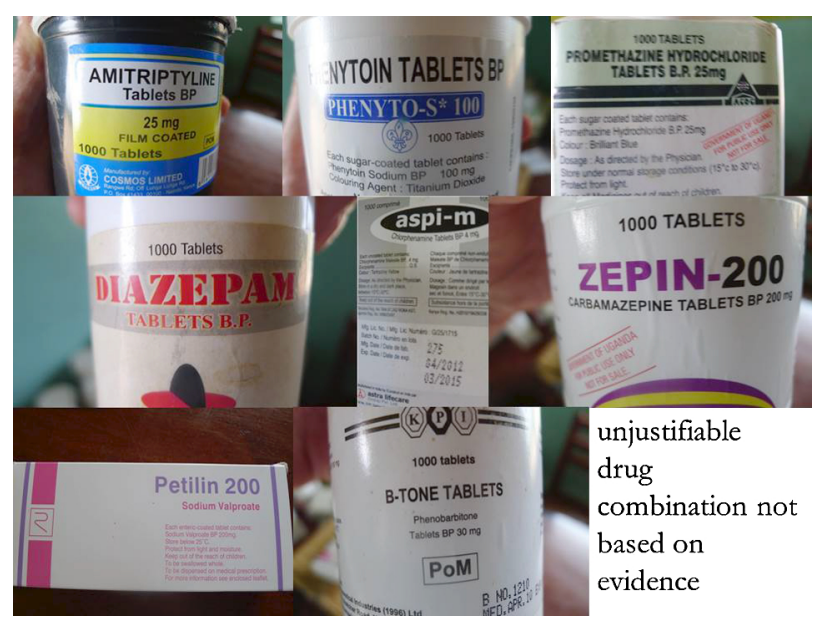

Fig. 4. Anti-epileptic drugs and vitamin B6 used in Uganda report that children still develop seizures.

\section{ReseARCH AgENDA}

In order to understand the natural history of the disease, to identify the cause(s) and to develop an effective case management the following steps are suggested (Table 12):

- description of the epidemic

- description of the natural history of the disease

Table 12. Research Agenda 1-4

1. Describe the epidemic

- geographic extension + spread

- number, age, sex, family relationships, ethnicity of NS patients

- date of onset of NS => epidemic curve

- surveillance

$$
=>\text { new cases? }=>\text { ongoing exposure ? }
$$

- relationship to civil war / camps

- temporal comparison with other outbreaks of NS (e.g. Tanzania)

2. Describe the natural history of NS

- symptoms and signs; order of appearance; typical patterns

- neurological investigations: EEG, visually evoked potentials, EMG, MRI; CSF; cognitive functions

- pediatric investigations: malnutrition, stunting, mental retardation, delayed puberty

- laboratory investigations: onchocerciasis, sleeping sickness, malaria; viral infections; immune status

$\rightarrow$ develop staging/classification

3. Identify cause(s)

- list potential causes+confounders

- create research consortium

- define investigations to be undertaken

- implement epidemiological studies in two districts / areas

\section{Ameliorate case management}

- personalize treatment based on evidence

- implement appropriate health infrastructure

- support affected families 


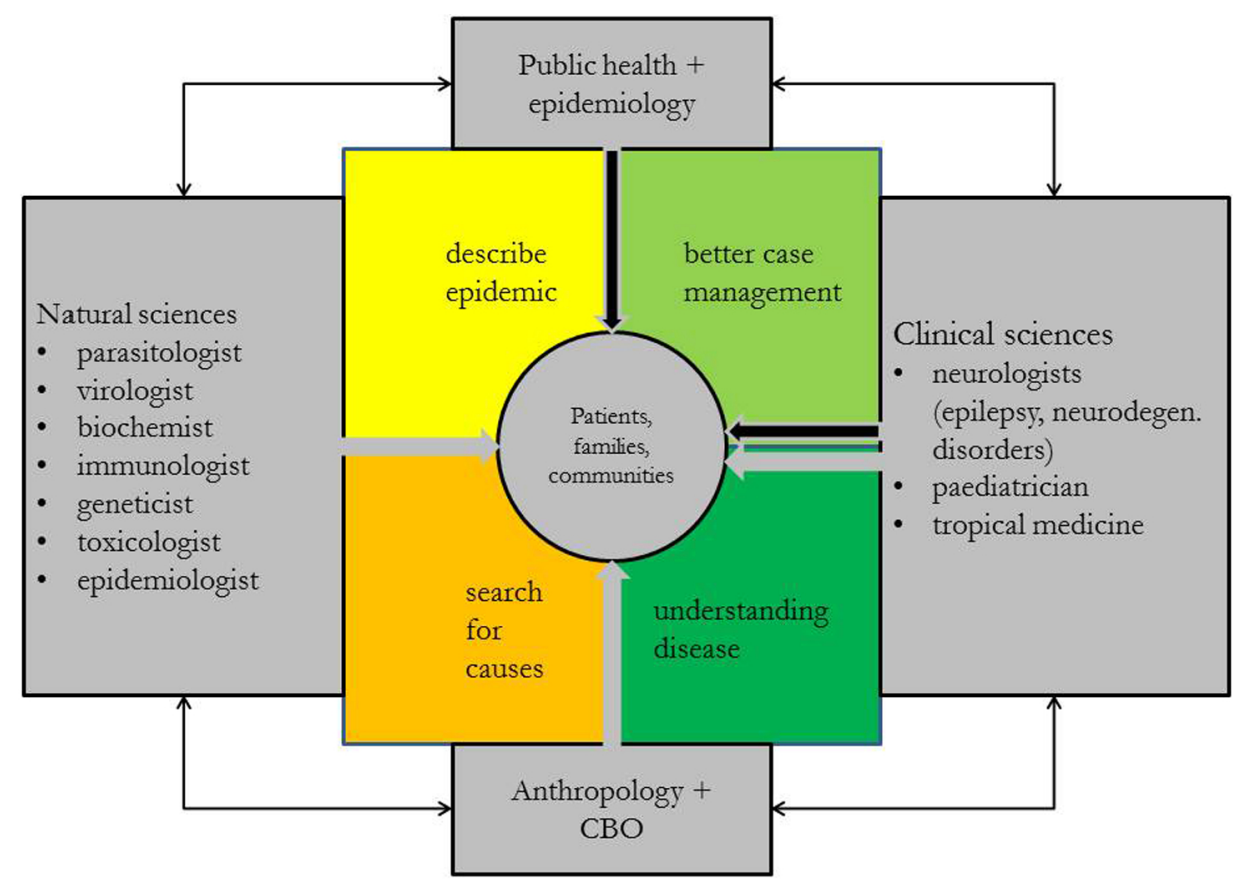

Fig. 5. Transdisciplinary approach to understand and control nodding syndrome

- identification of the cause(s) and understanding of the pathophysiology of nodding syndrome

- personalized treatment and support for affected families and communities

Obviously, nodding syndrome is a complex health issue and can only be solved through a transdisciplinary approach (Fig. 5).

\section{REFERENCES}

1. Jilek LA. Geisteskrankheiten und Epilepsie im tropischen Afrika. Fortschr Neurol Psychiatr Grenzgeb 1964; 32: 213-259 (in German).
2. Sevjar JJ, Kakooza AM, Foltz JL, Makumbi I, AtaiOmoruto AD, Malimbo M, Ndyomugyenyi R, Alexander LN, Abang B, Downing RG, Ehrenberg A, Guilliams K, Helmers S, Melstrom P, Olara D, Perlman S, Ratto J, Trevathan E, Winkler AS, Dowell SF, Lwamafa DKW. Clinical, neurological, and electrophysiological features of nodding syndrome in Kitgum, Uganda: an observational case series. Lancet Neurol 2013; 12: 166-174.

3. Dowell SF, Sejvar JJ, Riek L, Vandemaele KAH, Lamunu M, Kuesel AC, Schmutzhard E, Matuja W, Bunga S, Foltz JL, Nutman TB, Winkler AS, Mbonye K. Nodding Syndrome. Emerg Infect Dis 2013; 19: 1374-1384. 